\title{
Application of technology in meeting unmet needs of older people living in the community
}

\author{
Author: Jean Woo
}

Aims

To test the feasibility of screening for unmet needs of older people by multi-domain assessment, using a mobile device.

\section{Methods}

A brief multi-domain questionnaire consisting of 13 questions was constructed to elicit primary care needs of older people according to the World Health Organization (WHO) recommendations, covering sensory deficits, chewing problems, functional capacity, self-rated health, frailty, sarcopenia, memory impairment, incontinence, pharmacy, psychological wellbeing and financial security. Older people attending community centres were invited to participate in responding through an iPad. Answers were transmitted to a data centre in a tertiary academic unit. For each response indicator possible abnormality, a response pathway would provide further follow up, either further detailed assessments (such as cognitive function) or providing advice and information regarding local available resource (such as recommendation for frailty prevention group exercises, checking of drugs for polypharmacy, advice re use of aids where for problems with activities of daily living or instrumental activities of daily living).

\section{Results}

Over a 1-month period in 2016, 2,500 people were screened. This survey identified $75 \%$ with memory problems; $64 \%$ with varying levels of frailty; $38 \%$ with chewing difficulties; $14 \%$ with sarcopenia; $14 \%$ with difficulties in instrumental activities of daily living; $12 \%$ with poor self-rated health; and $10 \%$ who were not satisfied with life. All these problems are amenable to further assessments and action before referral to medical clinics if necessary.

The second phase is the automation of the further assessments and action pathways, to be used as a computer program or through the development of an app, and this would complement and aid the work of primary health care centres in meeting the needs of older people.

Author: Department of Medicine \& Therapeutics, Faculty of Medicine, The Chinese University of Hong Kong, Hong Kong

\section{Conclusion}

The findings support the development of technology in primary care of older people following the WHO's proactive approach emphasising maintaining intrinsic capacity (or prevention of frailty) in addition to targeting individual chronic diseases, by making use of technology in a step care approach in early detection of problems and guide to subsequent action that allows for management in the community reducing reliance on the hospital system.

\section{Conflict of interest statement}

None declared. 